\title{
Optical Guiding of Intense Hermite-Gaussian Laser Beam in Preformed Plasma Channel and Second Harmonic Generation
}

\section{Jyoti Wadhwa}

NIT Jalandhar: Dr BR Ambedkar National Institute of Technology

Arvinder Singh ( $\sim$ arvinders@nitj.ac.in )

Dr BR Ambedkar National Institute of Technology https://orcid.org/0000-0002-0628-7861

\section{Research Article}

Keywords: Hermite-Gaussian laser beam, Preformed plasma channel, Relativistic plasma, Second harmonic generation

Posted Date: February 11th, 2021

DOI: https://doi.org/10.21203/rs.3.rs-161791/v1

License: (c) (i) This work is licensed under a Creative Commons Attribution 4.0 International License.

Read Full License

Version of Record: A version of this preprint was published at Optical and Quantum Electronics on July 22nd, 2021. See the published version at https://doi.org/10.1007/s11082-021-03046-z. 


\title{
Optical guiding of intense Hermite-Gaussian laser beam in preformed plasma channel and second harmonic generation
}

\author{
Jyoti Wadhwa and Arvinder Singh \\ Department of Physics, National Institute of Technology Jalandhar, India \\ Email :- jyotiwadhwa16@gmail.com,arvinders@nitj.ac.in
}

\begin{abstract}
This work presents the scheme of optical channeling of the intense Hermite Gaussian laser beam and second-harmonic generation in plasma having the preformed plasma channel, where relativistic nonlinearity is operative. Excitation of the electron plasma wave at the incident beam frequency leads its coupling with the latter produces the second harmonics of the beam. For the formulation of differential equations for the beam waists of the Hermite Gaussian laser beam propagating through the channel, the method of moments has been used. The solutions of the coupled differential equations are found using Runge Kutta fourth-order numerical method. Perturbation theory has been applied to find the equation governing the excitation of electron plasma wave and hence the source term for the second-harmonic yield. It has been observed that the preformed plasma channel helps to optically guide the laser beam and enhances the efficiency of second-harmonic generation of various modes of the Hermite Gaussian laser beam in plasma.
\end{abstract}

Keywords: Hermite-Gaussian laser beam, Preformed plasma channel, Relativistic plasma, Second harmonic generation.

\section{Declarations}

\section{Funding}

The authors are thankful to the Ministry of Human Resources and Development (MHRD) of India for providing the necessary funds to carry out the research work.

\section{Conflicts of interest/Competing interests}

NA

\section{Availability of data and material}

The data that support the findings of this study are available within the article. 


\section{Code availability}

NA

\section{Introduction}

The study of optical guiding of the laser beam in plasma leads to the applications in fusion energy, higher harmonic generation (Gibbon 1997), laser wakefield accelerators (Clayton et al. 2010), and soft x-ray laser (Nakano et al. 1997). The propagating laser must be having a very high intensity for the successful realization of the above-mentioned applications. Moreover, high-intensity beams are readily available these days but the challenges like beam break up due to filamentation, defocusing due to ionization, and Raman scattering is always there in the way of self-focusing of the beam. These challenges can be overcome by the technique of optical guiding in which the laser beam does not create its own channel but is propagated in a preformed channel (Esarey and Sprangle 1997) These preformed channels are stable and reproducible which is the necessity for any real application.

Plasmas are at the core of fusion, a possible solution to one of the most formidable energy challenges faced by mankind today. Unlike other dielectric fibers for the guiding of the laser beam, plasma fibers are most reliable because it supports not only high-intensity beams but also the wavelengths of soft x-ray regions. The problem of laser diffraction in plasma can be addressed by using laser beam guiding and preformed plasma channels to maintain the required laser intensity over distances of many Rayleigh lengths. Also, in laser wakefield accelerators some form of optical guiding is very important to avoid the need for multistage acceleration (Sprangle et al. 1988).

Second-harmonic generation is an optical process of coherent radiations, dependent on the second-order intensity term in the expansion of polarization (Basov et al. 1979). The electric field of the laser beam should be strong enough to generate the second order radiation otherwise the first-order term will dominate. This way, one can think of the second harmonic signal as a perturbation in which two light frequencies are added up to give rise to second-harmonic radiation. In plasma, the second-harmonic radiation can be generated in many ways but the most common is via the excitation of electron plasma wave at the pump frequency. Its propagation in plasma gives important information about the plasma parameters like local electron density, expansion velocity, etc. Most importantly, second-harmonic radiation is very useful in 
the process of laser ignited fusion due to its deep penetration power in overdense regions of the same.

A vast literature on the cutting edge illustrations on the topics of the channeling of the laser beam in plasma (Ehrlich et al. 1996) as well as second-harmonic generation (Singh and Gupta 2016; Sharma et al. 2020) in plasmas is available. Durfee et al. (1995) demonstrated the two laser-pulse technique for the channeling of intense laser pulses over distances much greater than a Rayleigh length. The first pulse creates a breakdown spark in a gas target which results in the expansion of the hot plasma forming the channel to guide the second pulse. The propagation of an intense sinusoidal laser pulse, in a preformed plasma channel having a parabolic density profile, has been reported (Upadhyay et al. 2007). Salih and co-workers (2003) investigated the second-harmonic generation of the circularly polarized laser beam in a self-created magnetized plasma channel. Gupta et al. (2015) investigated the second-harmonic generation of the $q$ Gaussian laser beam in a preformed collisional plasma channel by considering the nonlinear absorption of the laser beam.

Most of the work in optical guiding of the laser beam and its effect on second-harmonic generation has been carried out by using the Gaussian intensity profile under the paraxial ray approximation. Recently, Kant and Thakur (2021) analysed the self-focusing of the Hermite-Gaussian chirped pulse laser in plasma under the influence of linear absorption by using the paraxial approximation theory. The impulse of the present work resides in investigating the effect of a preformed parabolic density channel on the optical guiding of highly intense Hermite Gaussian laser beam and the generation of second harmonics in plasma by the moment theory approach suggested by Lam et al. 1977.

This paper is structured in the following way: Sect. 2 deals with the optical guiding of the Hermite Gaussian laser beam by using the dielectric constant for the preformed plasma channel under relativistic nonlinearity. In Sect. 3 density perturbation term has via the excitation of electron plasma wave has been discussed following by Sect. 4 which describes the equation for second-harmonic yield. Sect. 5 contains the details of the results and the conclusions drawn from the present analysis are summarised in Sect. 6 . 


\section{Guiding of Hermite Gaussian laser beam in preformed plasma chan-} nel

Consider the propagation of intense circularly polarized Hermite Gaussian laser beam through plasma having preformed plasma density channel of parabolic form ${ }^{16}$ i.e. $n_{e}(r)=n_{e}(0)+$ $\Delta n_{e}\left(\frac{r}{r_{c h}}\right)^{2}$ where $n_{e}(0)$ is the electron density at the axis of the channel, $\Delta n_{e}=n_{e}\left(r_{c h}\right)-n_{e}(0)$ is the depth of the channel which means the electron density increases radially from $r=0$ to $r=r_{c h}$. Relativistic nonlinearity in the plasma due to non-uniform irradiance of the laser beam and the preformed plasma channel combinedly alter the dielectric function of plasma as:

$$
\epsilon=1-\frac{\omega_{p 0}^{2}}{\gamma \omega_{0}^{2}}-\frac{\omega_{p c h}^{2}}{\gamma \omega_{0}^{2}} \frac{r^{2}}{r_{c h}^{2}}
$$

where

$$
\begin{gathered}
\omega_{p 0}^{2}=\sqrt{\frac{4 \pi n_{e}(0) e^{2}}{m_{0}}}, \\
\omega_{p c h}^{2}=\sqrt{\frac{4 \pi \Delta n_{e}(0) e^{2}}{m_{0}}},
\end{gathered}
$$

are the corresponding plasma frequencies and

$$
\gamma=\sqrt{1+\beta E E^{*}}
$$

is the relativistic Lorentz factor with $\beta=\left(e^{2} / m_{0}^{2} \omega_{0}^{2} c^{2}\right)$ being the coefficient of relativistic nonlinearity. $E E^{*}$ is the intensity distribution function of the laser beam across its wavefront which is considered to be Hermite Gaussian ${ }^{17}$ and is used in the following form:

$$
E E^{*}=\frac{A_{0}^{2}}{f_{x} f_{y}} \exp \left(-\frac{x^{2}}{r_{0}^{2} f_{x}^{2}}-\frac{y^{2}}{r_{0}^{2} f_{y}^{2}}\right) H_{m}\left(\frac{x}{r_{0} f_{x}}\right) H_{n}\left(\frac{y}{r_{0} f_{y}}\right),
$$

where $A_{0}$ represents the maximum axial amplitude of the electric field of the laser beam, $r_{0} f_{x}$ and $r_{0} f_{y}$ represent the instantaneous beam waists in the transverse $x$ and $y$ directions respectively, and $H_{m}$ and $H_{n}$ are the corresponding Hermite polynomials. The intensity profile corresponding to mode $E_{00}$ of Hermite polynomial is Gaussian which is shown in Fig. 1 along with two other modes of Hermite Gaussian beam i.e. $E_{01}$ and $E_{02}$.

From Eq.(1), the revised dielectric function for plasma after the propagation of laser beam can be expressed in the form of intensity independent part i.e. $\epsilon_{0}$ and intensity-dependent part $\Phi\left(E E^{\star}\right)$ as:

$$
\epsilon=\epsilon_{0}+\Phi\left(E E^{\star}\right)
$$


where

$$
\begin{gathered}
\epsilon_{0}=1-\frac{\omega_{p 0}^{2}}{\omega_{0}^{2}}, \\
\Phi\left(E E^{\star}\right)=\frac{\omega_{p 0}^{2}}{\omega_{0}^{2}}-\left(\frac{\omega_{p 0}^{2}}{\omega_{0}^{2}}+\frac{\omega_{p c h}^{2}}{\omega_{0}^{2}} \frac{x^{2}+y^{2}}{r_{c h}^{2}}\right)\left(1+\beta E E^{\star}\right)^{-1 / 2},
\end{gathered}
$$

Under the W.K.B. approximation, the following wave equation is satisfied by the wave field vector of the laser beam

$$
\nabla^{2} \mathbf{E}+\frac{\omega_{0}^{2}}{c^{2}} \epsilon \mathbf{E}=0
$$

Substituting Eqs.(5) and (6) in Eq.(9) and assuming the slower variations in the $z$-direction than those in the transverse directions, the following quasi-optic wave equation is obtained

$$
i \frac{d E}{d z}=\frac{1}{2 k_{0}} \nabla_{\perp}^{2} E+\frac{k_{0}}{2 \epsilon_{0}} \Phi\left(E E^{\star}\right) E .
$$

According to Lam et al. ${ }^{14}$, the intensity and mean square radius of the laser beam which is defined by the zeroth and second-order spatial moments, are given by

$$
I_{0}=\iint E E^{\star} d x d y
$$

and

$$
<a^{2}>=\frac{\iint\left(x^{2}+y^{2}\right) E E^{\star} d x d y}{\iint E E^{\star} d x d y} .
$$

Differentiating Eq.(12) twice with respect to $z$ and using Eq.(10) we get

$$
\frac{d^{2}}{d z^{2}}<r_{r m s}^{2}>=4 \frac{R}{I_{0}}+4 \frac{H}{I_{0}},
$$

where

$$
\begin{gathered}
R=\iint\left(\left|\nabla_{\perp} E\right|^{2}-F\right) d x d y \\
F\left(E E^{\star}\right)=\frac{1}{2 \epsilon_{0}} \int_{0}^{E E^{\star}} \Phi\left(E E^{\star}\right) d\left(E E^{\star}\right),
\end{gathered}
$$

and

$$
H=\iint\left(2 F-\frac{1}{2 \epsilon_{0}} E E^{\star} \Phi\left(E E^{\star}\right)\right) d x d y .
$$

Substituting Eq.(5) in Eqs.(11) and (12) one can have,

$$
I_{0}=\pi A_{0}^{2} r_{0}^{2} m ! n ! 2^{m+n}
$$


and

$$
\begin{aligned}
<a^{2}> & =<a_{x}^{2}>+<a_{y}^{2}> \\
& =r_{0}^{2} f_{x}^{2}\left(m+\frac{1}{2}\right)+r_{0}^{2} f_{y}^{2}\left(n+\frac{1}{2}\right) .
\end{aligned}
$$

Differentiating Eq.(18) two times with respect to $z$ and using Eqs.(5) and (10-18), the following nonlinear coupled differential equations for the transverse beam waist parameters $f_{x}$ and $f_{y}$ in the $x$ and $y$ directions respectively are obtained:

$$
\begin{aligned}
& \frac{d^{2} f_{x}}{d \xi^{2}}+\frac{1}{f_{x}}\left(\frac{d f_{x}}{d \xi}\right)^{2}=\frac{1}{f_{x}^{3}}+\frac{1}{\pi} \frac{I_{1}}{\left(m+\frac{1}{2}\right) 2^{m+n-1} m ! n !} \\
& \frac{d^{2} f_{y}}{d \xi^{2}}+\frac{1}{f_{y}}\left(\frac{d f_{y}}{d \xi}\right)^{2}=\frac{1}{f_{y}^{3}}+\frac{1}{\pi} \frac{I_{2}}{\left(n+\frac{1}{2}\right) 2^{m+n-1} m ! n !}
\end{aligned}
$$

where

$$
\begin{gathered}
I_{1}=-2\left(\frac{\omega_{p c h}^{2} r_{0}^{2}}{c^{2}}\right)\left(\frac{r_{0}^{2}}{r_{c h}^{2}}\right) f_{x} I_{11}+\left(\frac{\omega_{p 0}^{2} r_{0}^{2}}{c^{2}}\right) \frac{\beta A_{0}^{2}}{f_{x}^{2} f_{y}} I_{12}+\left(\frac{\omega_{p c h}^{2} r_{0}^{2}}{c^{2}}\right)\left(\frac{r_{0}^{2}}{r_{c h}^{2}}\right) \frac{\beta A_{0}^{2}}{f_{x}^{2} f_{y}} I_{13}, \\
I_{2}=-2\left(\frac{\omega_{p c h}^{2} r_{0}^{2}}{c^{2}}\right)\left(\frac{r_{0}^{2}}{r_{c h}^{2}}\right) f_{y} I_{21}+\left(\frac{\omega_{p 0}^{2} a^{2}}{c^{2}}\right) \frac{\beta A_{0}^{2}}{f_{x} f_{y}^{2}} I_{22}+\left(\frac{\omega_{p c h}^{2} r_{0}^{2}}{c^{2}}\right)\left(\frac{r_{0}^{2}}{r_{c h}^{2}}\right) \frac{\beta A_{0}^{2}}{f_{x} f_{y}^{2}} I_{23}, \\
I_{11}=\int_{-\infty}^{\infty} \int_{-\infty}^{\infty} u^{2} e^{-\left(u^{2}+v^{2}\right)} H_{m}^{2}(u) H_{n}^{2}(v) K^{-1 / 2} d u d v, \\
I_{12}=\int_{-\infty}^{\infty} \int_{-\infty}^{\infty} e^{-2\left(u^{2}+v^{2}\right)} H_{m}^{3}(u) H_{n}^{4}(v)\left\{u^{2} H_{m}(u)-u H_{m+1}(u)\right\} K^{-3 / 2} d u d v, \\
\int_{-\infty}^{\infty} \int_{-\infty}^{\infty}\left(f_{x}^{2} u^{2}+f_{y}^{2} v^{2}\right) e^{-2\left(u^{2}+v^{2}\right)} H_{m}^{3}(u) H_{n}^{4}(v)\left\{u^{2} H_{m}(u)-u H_{m+1}(u)\right\} K^{-3 / 2} d u d v, \\
I_{22}=\int_{-\infty}^{\infty} \int_{-\infty}^{\infty} e^{-2\left(u^{2}+v^{2}\right)} H_{m}^{4}(u) H_{n}^{3}(v)\left\{v^{2} H_{n}(v)-v H_{n+1}(v)\right\} K^{-3 / 2} d u d v, \\
I_{23}=\int_{-\infty}^{\infty} \int_{-\infty}^{\infty}\left(f_{x}^{2} u^{2}+f_{y}^{2} v^{2}\right) e^{-2\left(u^{2}+v^{2}\right)} H_{m}^{4}(u) H_{n}^{3}(v)\left\{v^{2} H_{n}(v)-v H_{n+1}(v)\right\} K^{-3 / 2} d u d v, \\
K=\left(1+\frac{\beta A_{0}^{2}}{f_{x} f_{y}} H_{m}^{2}(u) H_{n}^{2}(v) e^{-\left(u^{2}+v^{2}\right)}\right), \\
u=\frac{x}{a f_{x}}, \\
v=\frac{y}{b f_{y}},
\end{gathered}
$$

and, $\xi=z / k_{0} r_{0}^{2}$ being the dimensionless distance of propagation. The boundary conditions $f_{x, y}=1$ and $d f_{x, y} / d \xi=0$ at $\xi=0$ are applied to the Eqs.(19) and (20) which serve as the basic equations to study the propagation dynamics of Hermite-Gaussian laser beam in preformed plasma channel under the effect of relativistic nonlinearity. Numerical simulations to solve the same have been carried out with the aid of the RK $4^{\text {th }}$ order method. 


\section{Density perturbation term}

The electric field of a highly intense laser beam accelerates the mobile plasma electrons until the relatively stationary ions pull them back via the Coulomb force. The resulting electron plasma wave propagates in the wake of the incident beam with the same frequency. Following the fluid equations and the linear perturbation theory, one can obtain the following equation for the perturbed density of plasma which results in the excitation of electron plasma wave having the same frequency as that of the incident beam,

$$
-\omega_{0}^{2} n_{1}+v_{t h}^{2} \nabla^{2} n_{1}+\frac{1}{\gamma}\left(\omega_{p 0}^{2}+\omega_{p c h}^{2} \frac{r^{2}}{r_{c h}^{2}}\right)=-\frac{e}{m} n_{0} \nabla E
$$

where $v_{t h}^{2}=2 K_{B} T_{0} / m_{0}$ is the thermal velocity of electrons. Taking $n_{1} \propto e^{\iota\left(k_{0} z-\omega_{0} t\right)}$ in the above equation, one can have the following density perturbation term:

$$
n_{1}=-\frac{e n_{0}}{m_{0}} \frac{\nabla E}{\left\{\omega_{0}^{2}-k_{0}^{2} v_{t h}^{2}-\frac{1}{\gamma}\left(\omega_{p 0}^{2}+\omega_{p c h}^{2} \frac{r^{2}}{r_{c h}^{2}}\right)\right\}} .
$$

\section{Second harmonic yield}

The electron plasma wave having the same frequency as that of incident Hermite Gaussian laser beam constructively superimpose on the later and give rise to the generation of second-harmonic radiation which satisfies the following wave equation for the electric field vector $E_{2}$

$$
\nabla^{2} E_{2}+\frac{\omega_{2}^{2}}{c^{2}} \epsilon_{2}\left(\omega_{2}\right) E_{2}=\frac{4 \pi e^{2}}{c^{2}} n_{1} E,
$$

where $\epsilon_{2}$ is the dielectric constant of the plasma generated second-harmonic radiation and $\omega_{2}=2 \omega_{0}$ is the corresponding frequency. For $E_{2} \propto E_{2} e^{\iota\left(k_{2} z-\omega_{2} t\right)}$, the second harmonic electric field vector is solved as

$$
E_{2}=\frac{\omega_{p 0}^{2}}{c^{2}} \frac{n_{1}}{n_{0}} \frac{E}{\left(k_{2}^{2}-4 k_{0}^{2}\right)}
$$

The normalized effeciency or yield of second-harmonic radiation in plasma is defined as the quotient of second-harmonic radiation power to the power of incident laser beam.

$$
Y=\frac{\int_{-\infty}^{\infty} \int_{-\infty}^{\infty} E_{2} E_{2}^{\star} d x d y}{\int_{-\infty}^{\infty} \int_{-\infty}^{\infty} E E^{\star} d x d y}
$$

On substituting Eqs.(5), (22) and (24) in (25), the following equation of second-harmonic yield (Y) for the intense Hermite-Gaussian laser beam in the preformed plasma channel is obtained

$$
Y=\frac{1}{9 \pi} \frac{\beta A_{0}^{2}}{f_{x} f_{y}} \frac{\left(\frac{\omega_{0}^{2} a^{2}}{c^{2}}\right)}{\left(\frac{\omega_{p 0}^{2} a^{2}}{c^{2}}\right)} \frac{I_{3}}{m ! n ! 2^{m+n}},
$$


where

$$
\begin{aligned}
& I_{3}=\int_{-\infty}^{\infty} \int_{-\infty}^{\infty} e^{-\left(u^{2}+v^{2}\right)} H_{m}^{2}(u) H_{n}^{2}(v)\left(\frac{\omega_{p 0}^{2} r_{0}^{2}}{c^{2}}+\frac{\omega_{p c h}^{2} r_{0}^{2}}{c^{2}} \frac{r_{0}^{2}}{r_{c h}^{2}}\left(f_{x}^{2} u^{2}+f_{y}^{2} v^{2}\right)\right)\left\{H_{m}^{2}(u) H_{n}^{2}(v)\left(\frac{u^{2}}{f_{x}^{2}}+\frac{v^{2}}{f_{y}^{2}}\right)\right. \\
& \left.+\frac{1}{f_{x}^{2}} H_{m+1}^{2}(u) H_{n}^{2}(v)+\frac{1}{f_{y}^{2}} H_{m}^{2}(u) H_{n+1}^{2}(v)-2 \frac{u}{f_{x}^{2}} H_{m}(u) H_{m+1}(u) H_{n}^{2}(v)-2 \frac{v}{f_{y}^{2}} H_{m}^{2}(u) H_{n}(v) H_{n+1}(v)\right\} \\
& \times \frac{1}{\left\{\frac{\omega_{0}^{2} a^{2}}{c^{2}}-\left(\frac{\omega_{0}^{2} a^{2}}{c^{2}}-\frac{\omega_{p 0}^{2} a^{2}}{c^{2}}\right) \frac{v_{t h}^{2}}{c^{2}}-\left(\frac{\omega_{p 0}^{2} r_{0}^{2}}{c^{2}}+\frac{\omega_{p c h}^{2} r_{0}^{2}}{c^{2}} \frac{r_{0}^{2}}{r_{c h}^{2}}\left(f_{x}^{2} u^{2}+f_{y}^{2} v^{2}\right)\right) K(u, v)^{-\frac{1}{2}}\right\}^{2}} d u d v .
\end{aligned}
$$

\section{Discussion of Results}

On solving the second-order coupled differential Eqs.(19) and (20) numerically by using the RK $4^{\text {th }}$ order method, one can have the beam waists $f_{x}$ and $f_{y}$ of the intense Hermite Gaussian laser beam having $E_{m n}$ modes respectively, in a plasma having preformed plasma channel. Further, the effect of the beam waists and the preformed channel on the second-harmonic generation is given in the form of the second-harmonic yield by equation (26). For solving the above-mentioned equations, the normalized intensity of the laser beam and normalized plasma density is taken as $\beta A_{0}^{2}=1, \frac{\omega_{p 0}^{2} r_{0}^{2}}{c^{2}}=14$, while other set of parameters are:

$$
\begin{aligned}
& \omega_{0}=1.78 \times 10^{15} \mathrm{rad} / \mathrm{sec} ; \\
& r_{0}=15 \mu \mathrm{m} ; \\
& r_{c h}=150 \mu \mathrm{m} ; \\
& T_{0}=10^{6} \mathrm{~K} .
\end{aligned}
$$

Figures 2 and 3 depict the oscillatory focusing of beam waists $f_{x}$ and $f_{y}$ in the transverse $x$ and $y$ directions with the dimensionless distance of propagation $\xi$ for different modes of propagation of Hermite Gaussian beams i.e. $E_{00}, E_{01}$, and $E_{02}$ respectively in the guided plasma. For $E_{00}$ mode i.e. fundamental Gaussian mode (Fig. 1a), $f_{x}$ and $f_{y}$ show similar focusing behavior because of the symmetrical intensity distribution in both directions. The Peak intensity distribution is Gaussian in the $\mathrm{x}$-direction for all the three modes but has different distribution in the $\mathrm{y}$-direction. In the case of $E_{01}$ mode, $f_{x}$, as well as $f_{y}$, observe less focusing as compared to $E_{00}$ mode. This can be explained from Figs. 1c-d which shows the null intensity for $E_{01}$ mode along the y-direction at the axis and the intensity is distributed in the off axial parts. Axial rays have a greater tendency to focus as compared to the off-axial rays. Therefore, $f_{x}$ and $f_{y}$ observe less focusing on this mode. In the case of $E_{02}$ mode, peak intensity is high as compared to $E_{00}$ and $E_{01}$ modes and there is small intensity on the axial region of the y-axis also. Hence $f_{x}$ observes maximum focusing for this mode and $f_{y}$ observes relatively less focusing as compared to the $E_{00}$ mode. 
Figures 4 and 5 show the variation of dimensionless beam waists $f_{x}$ and $f_{y}$ against the dimensionless distance of propagation $\xi$ for $E_{02}$ intensity distribution of Hermite Gaussian laser beam for different

values of normalized channel depth i.e. $\left(\frac{\omega_{p c h}^{2} r_{0}^{2}}{c^{2}}\right)\left(\frac{r_{0}^{2}}{r_{c h}^{2}}\right)=0,0.60,1.20$. It is observed from the figures that with no preformed plasma channel, there occurs less focusing of $f_{x}$ and complete defocusing of the beam occurs in the y-direction. On increasing the channel depth (i.e. 0.60) there is an increase in the extent of self-focusing in the x-direction and $f_{y}$ also shows self-focusing behavior. Therefore, the beam is now self-guided under the effect of the preformed plasma channel. On further increasing the value of normalized channel depth (i.e. 1.20) the non-linear refraction term dominates the natural diffraction of the laser beam, which leads to an increase in the extent of self-focusing of the beam in both directions. It is further observed that there is a decrease in the focusing length.

Figure 6 represents the effect of self-focusing of the Hermite Gaussian laser beam with different modes $E_{m n}$ on the second-harmonic generation of the beam with fixed parameters of normalized laser intensity, plasma density, and channel depth. It is observed that in the presence of a preformed plasma channel the second harmonic yield is maximum for $E_{02}$ mode of the Hermite Gaussian laser beam and least for $E_{01}$ mode. This is since harmonic yield depends on the beam waists in both the transverse $\mathrm{x}$ and $\mathrm{y}$ directions. Beam width parameter $f_{x}$ undergoes maximum self-focusing for $E_{02}$ mode, which dominates the effect of $f_{y}$ and hence give maximum yield for $E_{02}$ mode. Also, if $f_{x}$ and $f_{y}$ are oscillatory, then yield will be oscillatory as well, giving rise to a step-like behavior at the focal spots of the laser beam of high intensity.

Figure 7 represents the effect of normalized channel depth on the second harmonic yield. From figures 4 and 5 it is very clear that the presence of a preformed plasma channel enhances the selffocusing of the laser beam in both directions. With an increase in the self-focusing of the beam (on increasing the channel depth) the transverse density gradient becomes very steep at the focusing points, which enhances the amplitude of plasma wave and hence the second-harmonic yield.

\section{Conclusions}

In the present work, numerical simulations have been carried out for the optical guiding as well as the second-harmonic generation of the intense Hermite Gaussian laser beam in the preformed plasma channel using the method of moments. It is found that the $E_{02}$ mode of the Hermite Gaussian laser beam is more prominent for self focusing as well as second-harmonic generation in plasma as compared to the $E_{01}$ mode and the Gaussian mode i.e. $E_{00}$. The second main outcome of this work is that the presence of the preformed plasma channel greatly enhances the guiding and second-harmonic yield in the plasma for the Hermite Gaussian laser beam. Outcomes of the present research may be beneficial in the experimental research pertaining to the successful realization of laser-induced fusion process. 


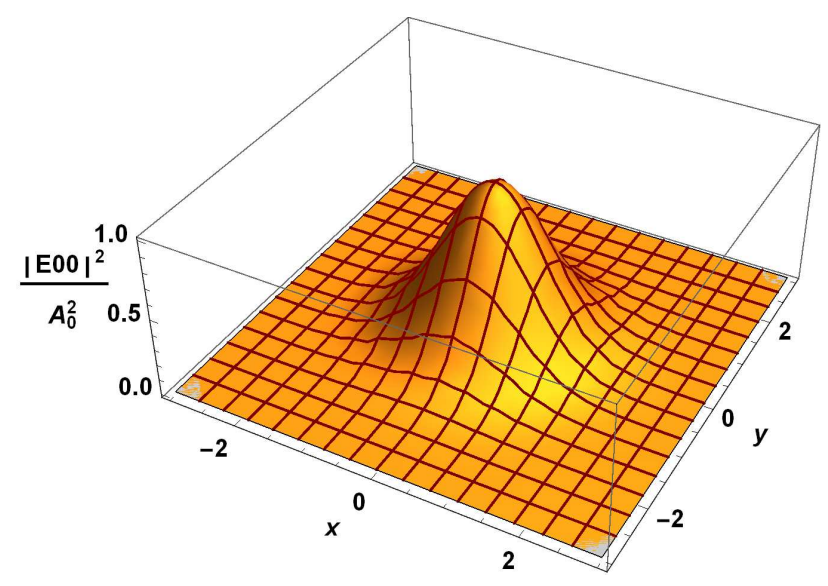

(a)

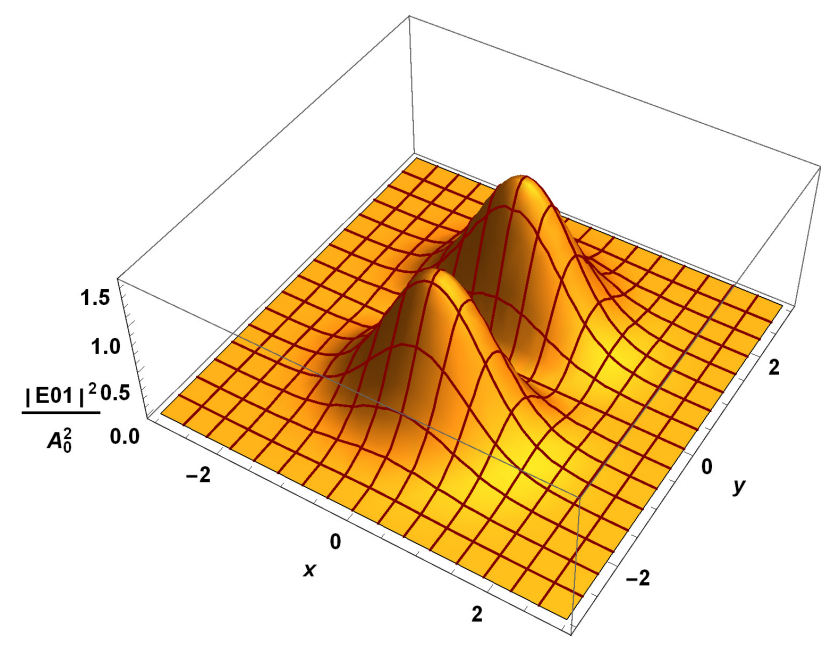

(c)

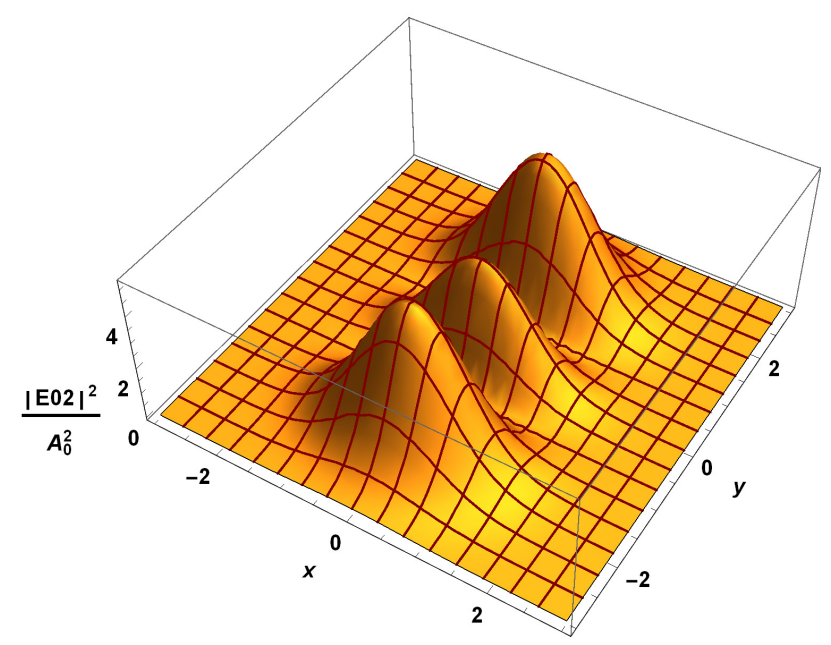

(e)

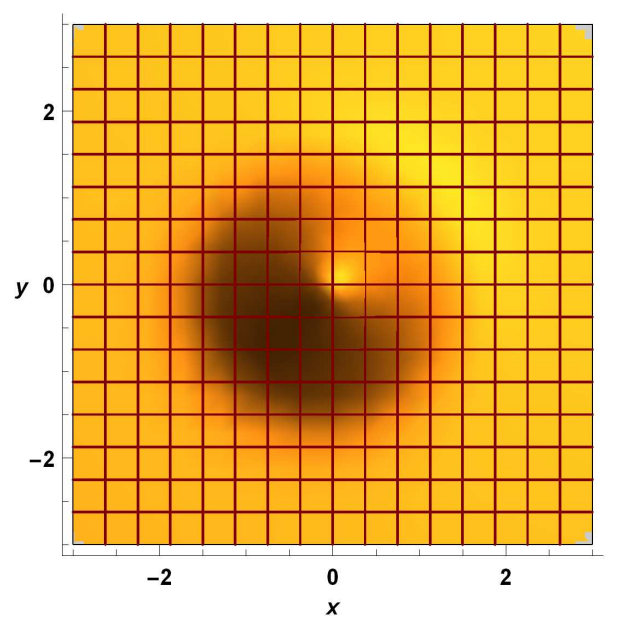

(b)

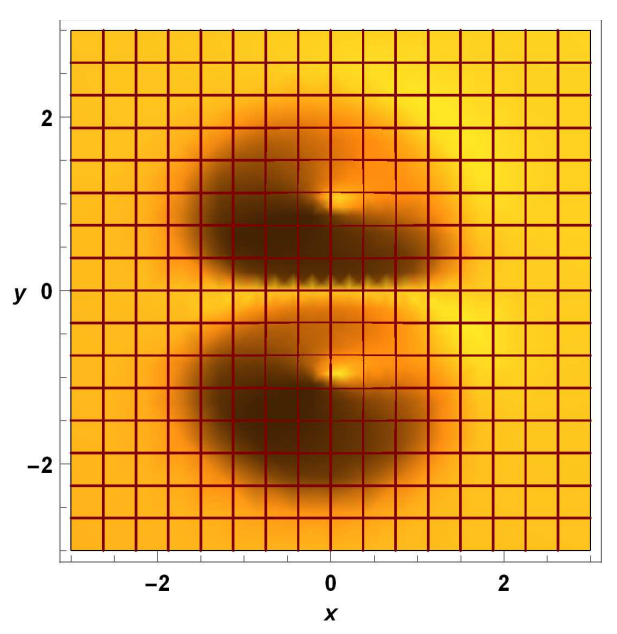

(d)

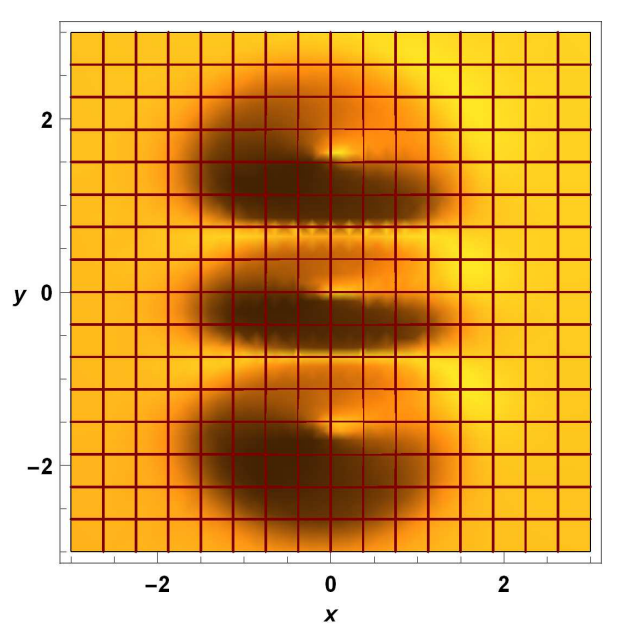

(f)

Figure 1: 3D intensity variation of Hermite Gaussian laser beam with the transverse $\mathrm{x}$ and $\mathrm{y}$-axis for different modes (a) $E_{00}$, (c) $E_{01}$, (e) $E_{03}$ and (b), (d), (f) represent the corresponding $x-y$ views. 


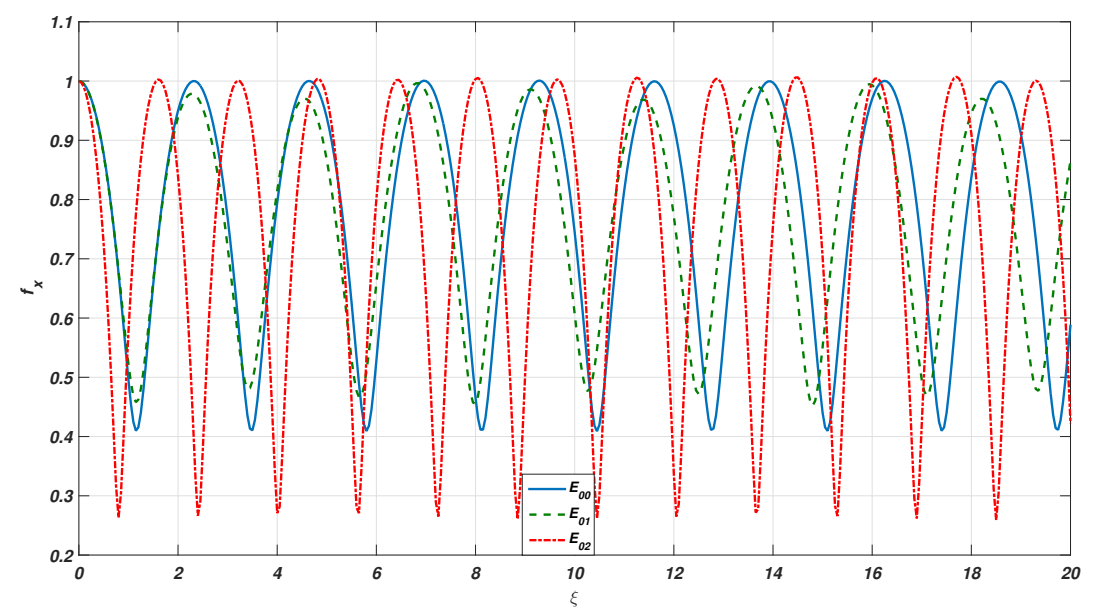

Figure 2: Variation of transverse beam waist parameter $f_{x}$ with the dimensionless distance of propagation $\xi=z / k_{0} r_{0}^{2}$ for different $E_{m n}$ modes and the normalized channel depth $\left(\omega_{p c h}^{2} r_{0}^{2} / c^{2}\right)\left(r_{0}^{2} / r_{c h}^{2}\right)=$ 0.60 .

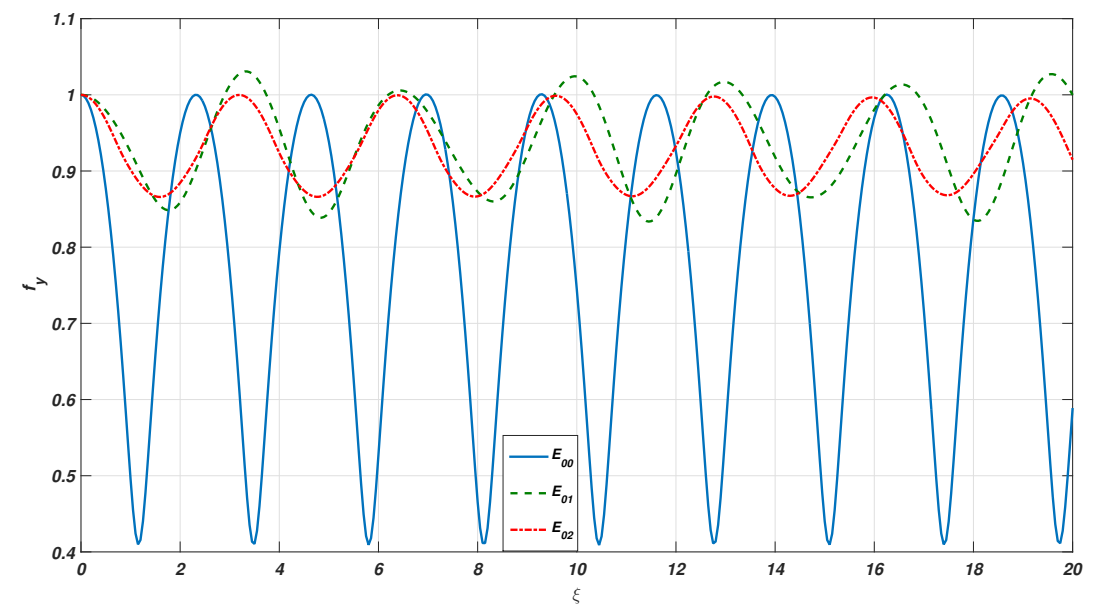

Figure 3: Variation of transverse beam waist parameter $f_{y}$ with the dimensionless distance of propagation $\xi=z / k_{0} r_{0}^{2}$ for different $E_{m n}$ modes and the normalized channel depth $\left(\omega_{p c h}^{2} r_{0}^{2} / c^{2}\right)\left(r_{0}^{2} / r_{c h}^{2}\right)=$ 0.60 . 


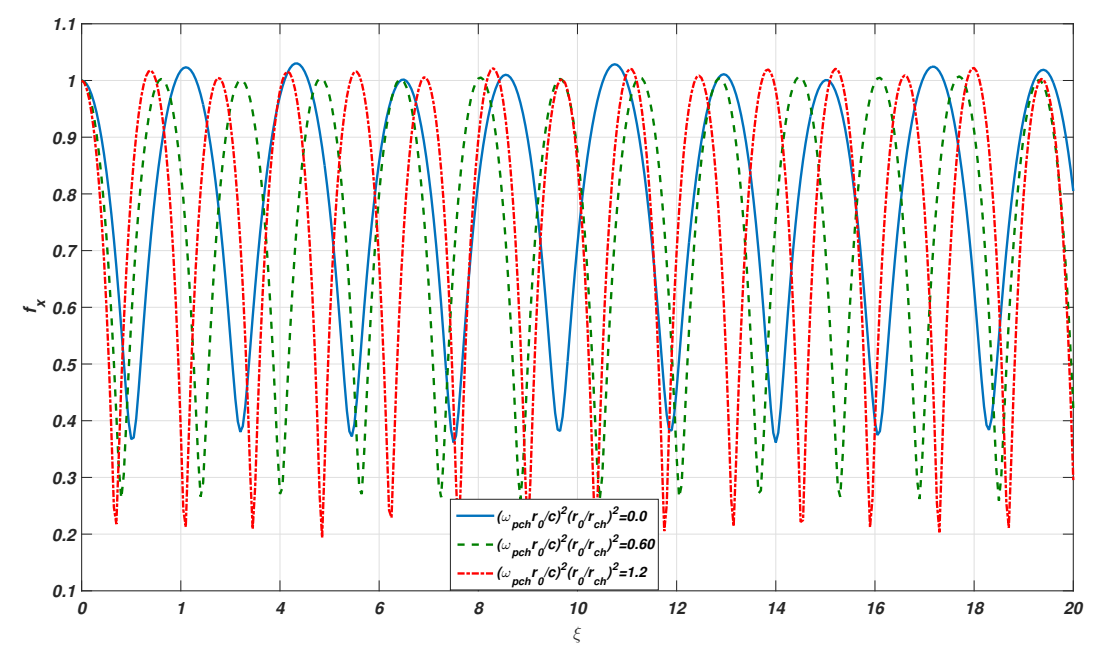

Figure 4: Variation of transverse beam waist parameter $f_{x}$ of $E_{02}$ mode of Hermite Gaussian laser beam with the dimensionless distance of propagation $\xi=z / k_{0} r_{0}^{2}$ for different values of the normalized channel depth.

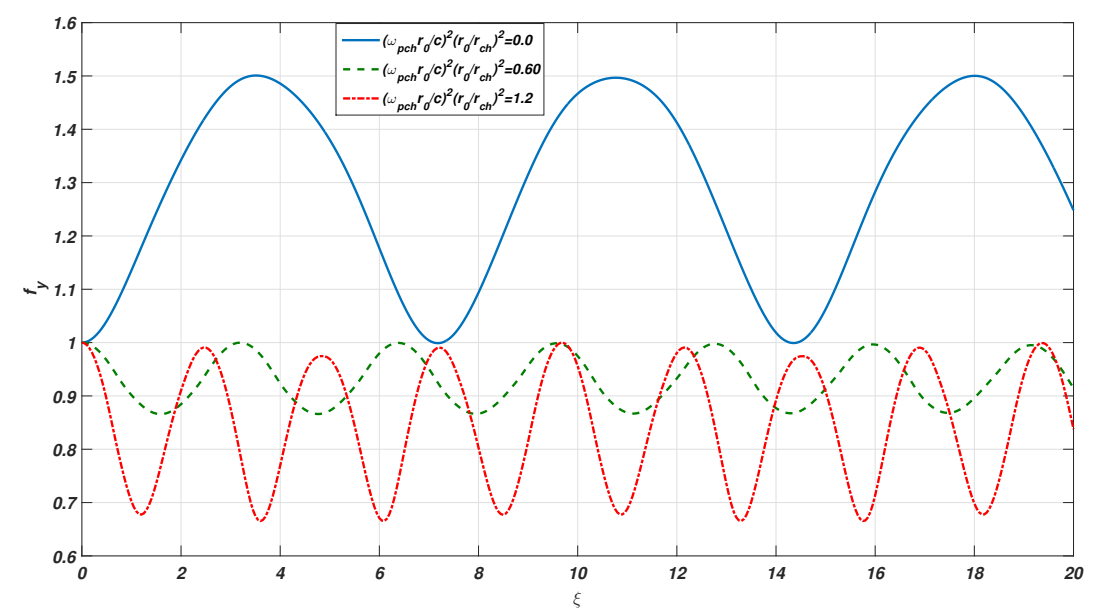

Figure 5: Variation of transverse beam waist parameter $f_{y}$ of $E_{02}$ mode of Hermite Gaussian laser beam with the dimensionless distance of propagation $\xi=z / k_{0} r_{0}^{2}$ for different values of the normalized channel depth. 


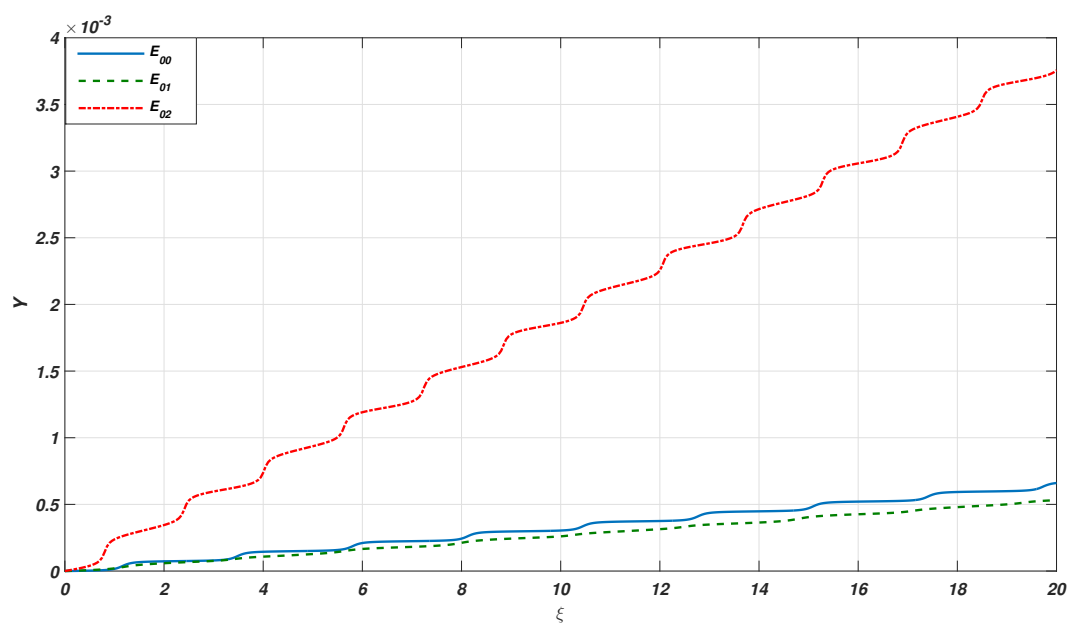

Figure 6: Variation of second harmonic yield $Y$ with the dimensionless distance of propagation $\xi=z / k_{0} r_{0}^{2}$ for different $E_{m n}$ modes and the normalized channel depth $\left(\omega_{p c h}^{2} r_{0}^{2} / c^{2}\right)\left(r_{0}^{2} / r_{c h}^{2}\right)=0.60$.

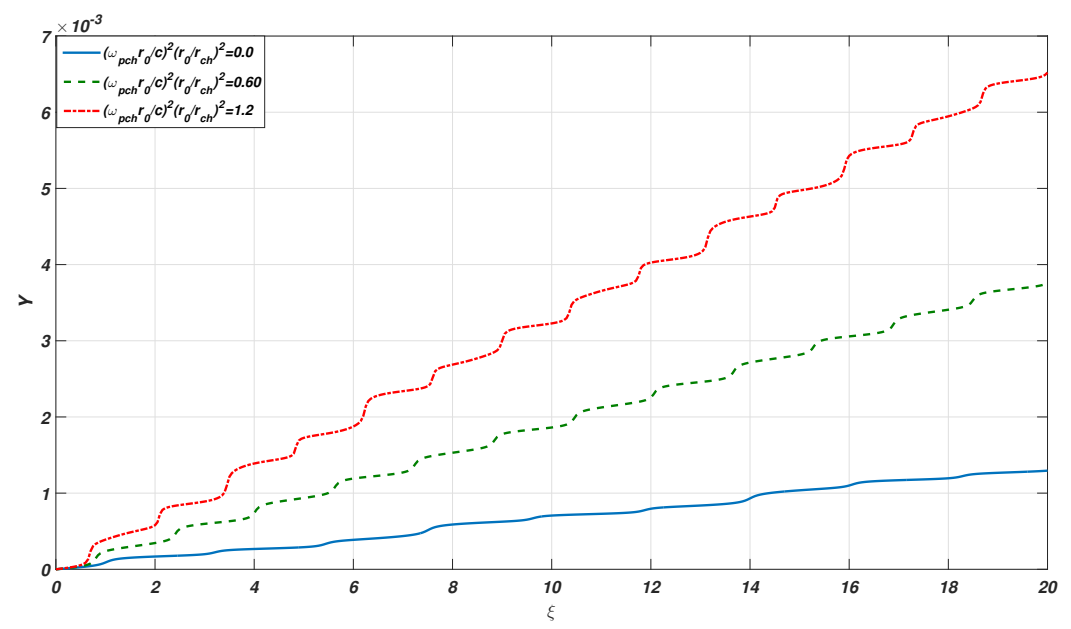

Figure 7: Variation of second harmonic yield $Y$ of $E_{02}$ mode of Hermite-Gaussian beam with the dimensionless distance of propagation $\xi=z / k_{0} r_{0}^{2}$ for different values of the normalized channel depth. 


\section{Refrences}

Basov, N.G., Bychenkov, V.Yu., Krokhin, O.N., Osipov, M.V., Rupasov, A.A., Silin, V.P., Sklizkov, G.V., Starodub, A.N., Tikhonchuk, V.T., Shikanov, A.S.: Second harmonic generation in a laser plasma (review). Soviet Journal of Quantum Electronics 9, 1081-1102 (1979)

Clayton, C.E., Ralph, J.E., Albert, F., Fonseca, R.A., Glenzer, S.H., Joshi, C., Lu, W., Marsh, K.A., Martins, S.F., Mori, W.B., Pak, A., Tsung, F.S., Pollock, B.B., Ross, J.S., Silva, L.O., Froula, D.H.: Self-Guided Laser Wakefield Acceleration beyond $1 \mathrm{GeV}$ Using Ionization-Induced Injection. Phys. Rev. Lett. 105, 105003 (2010)

Durfee, C.G., Lynch, J., Milchberg, H.M.: Development of a plasma waveguide for high-intensity laser pulses. Phys. Rev. E 51, 2368 (1995)

Ehrlich, Y., Cohen, C., Zigler, A.: Guiding of High Intensity Laser Pulses in Straight and Curved Plasma Channel Experiments. Phys. Rev. Lett. 77, 4176 (1996)

Esarey, E., Sprangle, P.: Self-focusing and guiding of short laser pulses in ionizing gases and plasmas. IEEE journal of quantum electronics 33, 1879 (1997)

Gibbon, P.: High-Order Harmonic Generation in Plasma. IEEE Journal of Quantum Electronics 33, 1915-1924 (1997)

Gupta, N., Singh, N., Singh, A.: Second harmonic generation of $q$-Gaussian laser beam in preformed collisional plasma channel with nonliner absorption nonlinear absorption. Phys. Plasmas 22, 113106 $(2015)$

Kant, N., Thakur, V.: Influence of linear absorption and density ramp on self-focusing of the HermiteGaussian chirped pulse laser in plasma. Opt. Quant. Electron. 53, 12(1-10) (2021)

Lam, J.F., Lippmann, B., Tappert, F.: Self-trapped laser beams in plasma. Phys. Fluids 20, 1176-1179 (1977)

Nakano, H., Nishikawa, T., Uesugi, N.: Soft x-ray pulse generation from femtosecond laser-produced plasma with reduced debris using a metal-doped glass target. Appl. Phys. Lett. 70, 16 (1997) 
Salih, H.A., Tripathi, V.K., Pandey, B.K.: Second-Harmonic Generation of a Gaussian Laser Beam in a Self Created Magnetized Plasma Channel. IEEE Trans. Plasma Sci. 31, 324 (2003)

Sharma, V., Thakur, V., Kant, N.: Second harmonic generation of cosh-Gaussian laser beam in magnetized plasma. Opt. Quant. Electron. 52, 444 (2020) doi:10.1007/s11082-020-02559-3

Singh, A., Gupta, N.: Second-harmonic generation by relativistic self-focusing of cosh-Gaussian laser beam in underdense plasma. Laser Part. Beams 34, 1-10 (2016)

Spence, D.J, Butler, A., Hooker, S.M.: First demonstration of guiding of high-intensity laser pulses in a hydrogen-filled capillary discharge waveguide. J. Phys. B: At. Mol. Opt. Phys. 34, 4103-4112 (2001)

Sprangle, P., Esarey, E., Ting, A., Joyce, G.: Laser wakefield accelration and relativistic optical guiding. Appl. Phys. Lett. 53, 2146 (1988)

Upadhyay, A.K., Raj, G., Mishra, R.K., Jha, P.: Propagation of sinusoidal pulse laser beam in a plasma channel. Phys. plasmas 14, 113105 (2007)

Wadhwa, J., Singh, A.: Generation of second harmonics of intense Hermite-Gaussian laser beam in relativistic plasma. Laser and Particle Beams 37, 79-85 (2019) 
Figures

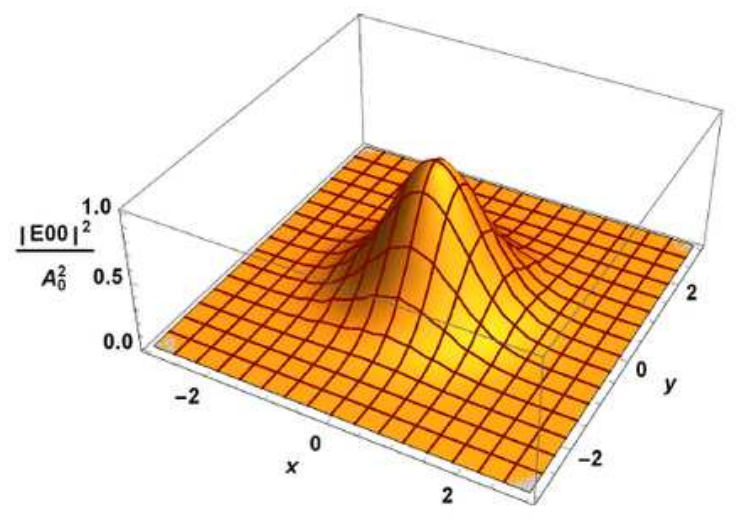

(a)

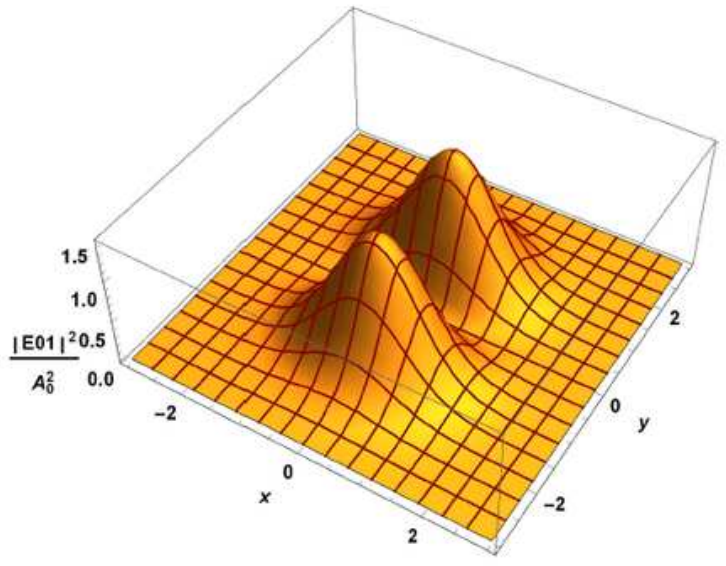

(c)

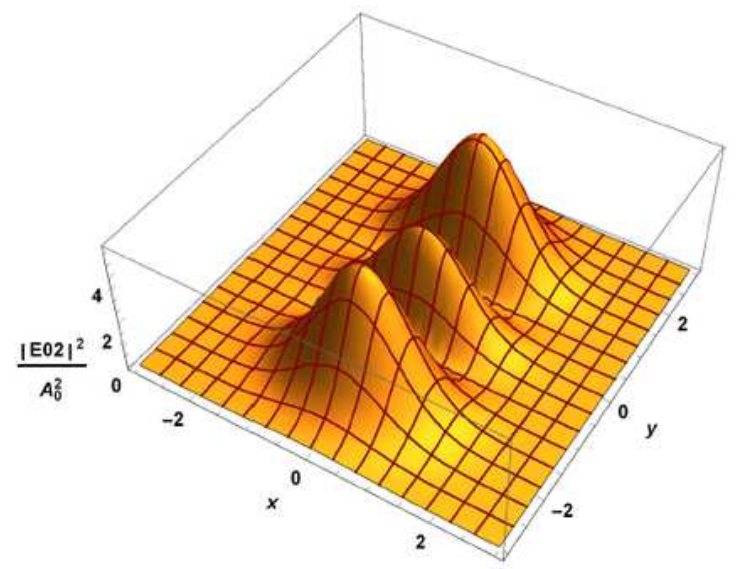

(e)

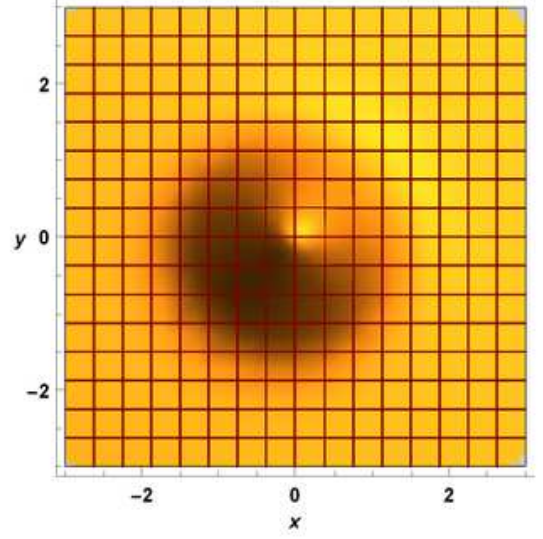

(b)

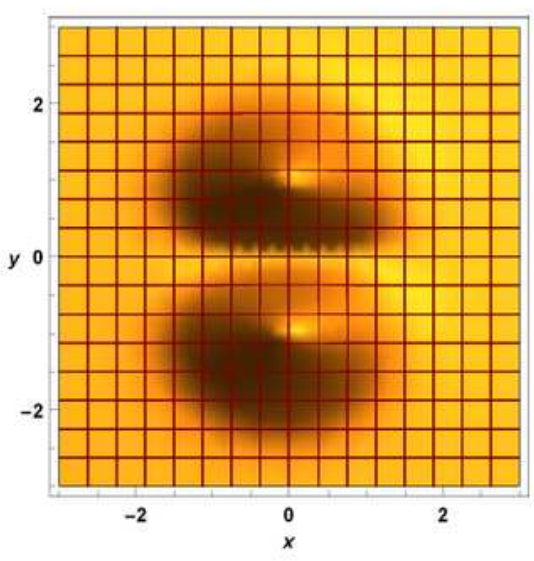

(d)

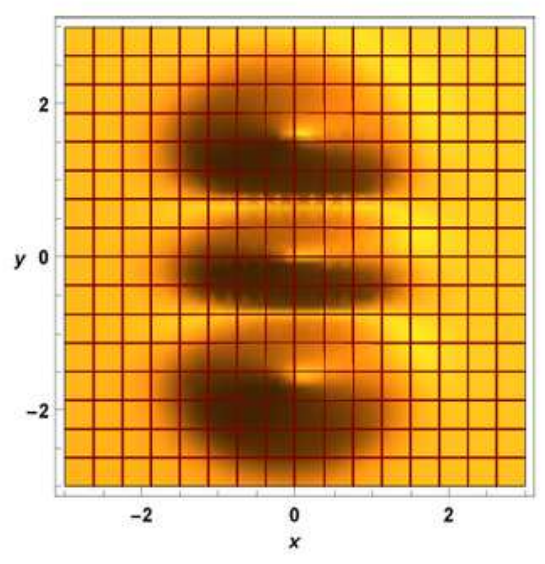

(f)

\section{Figure 1}

See the Manuscript Files section for the complete figure caption. 


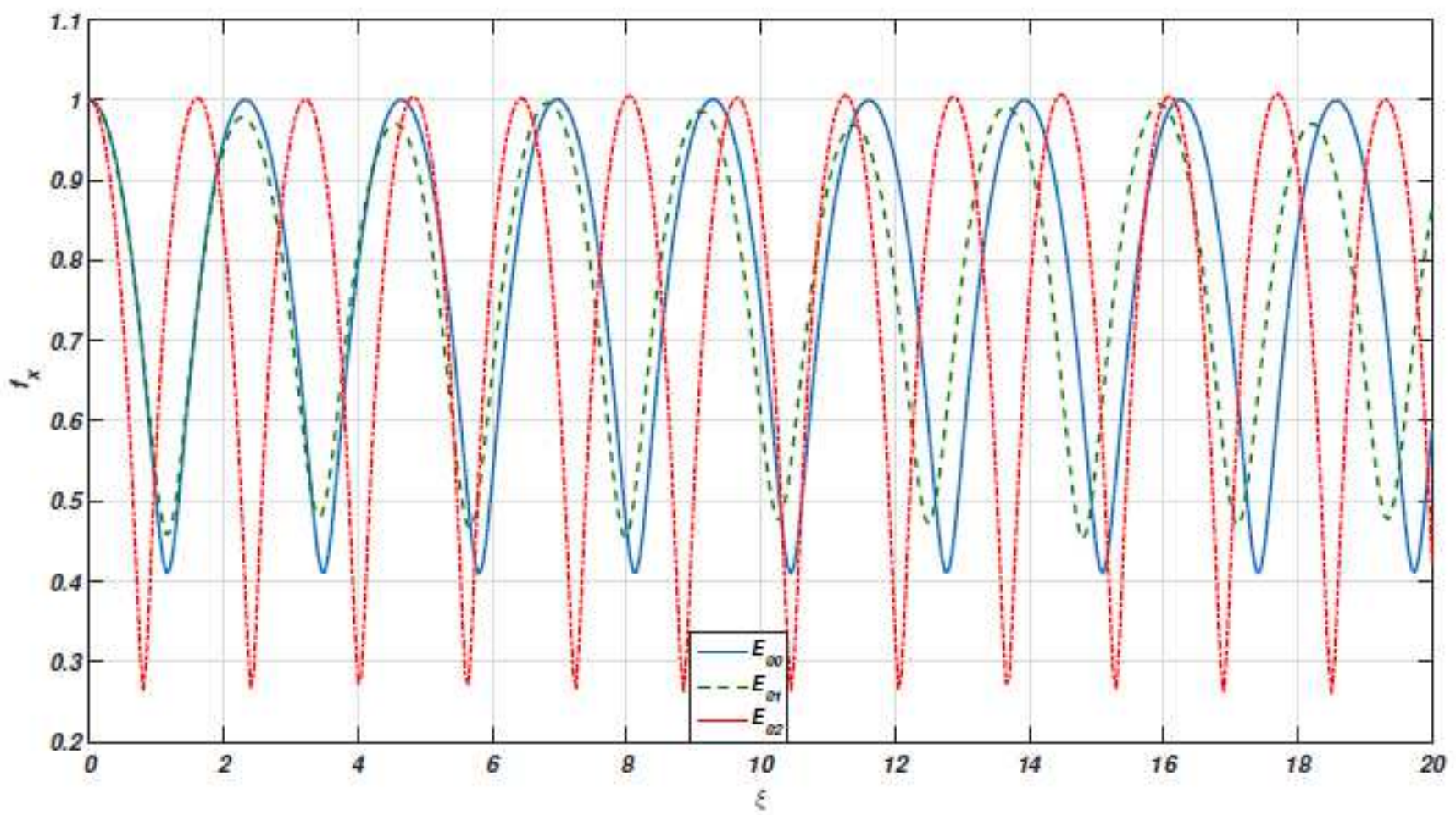

Figure 2

See the Manuscript Files section for the complete figure caption.

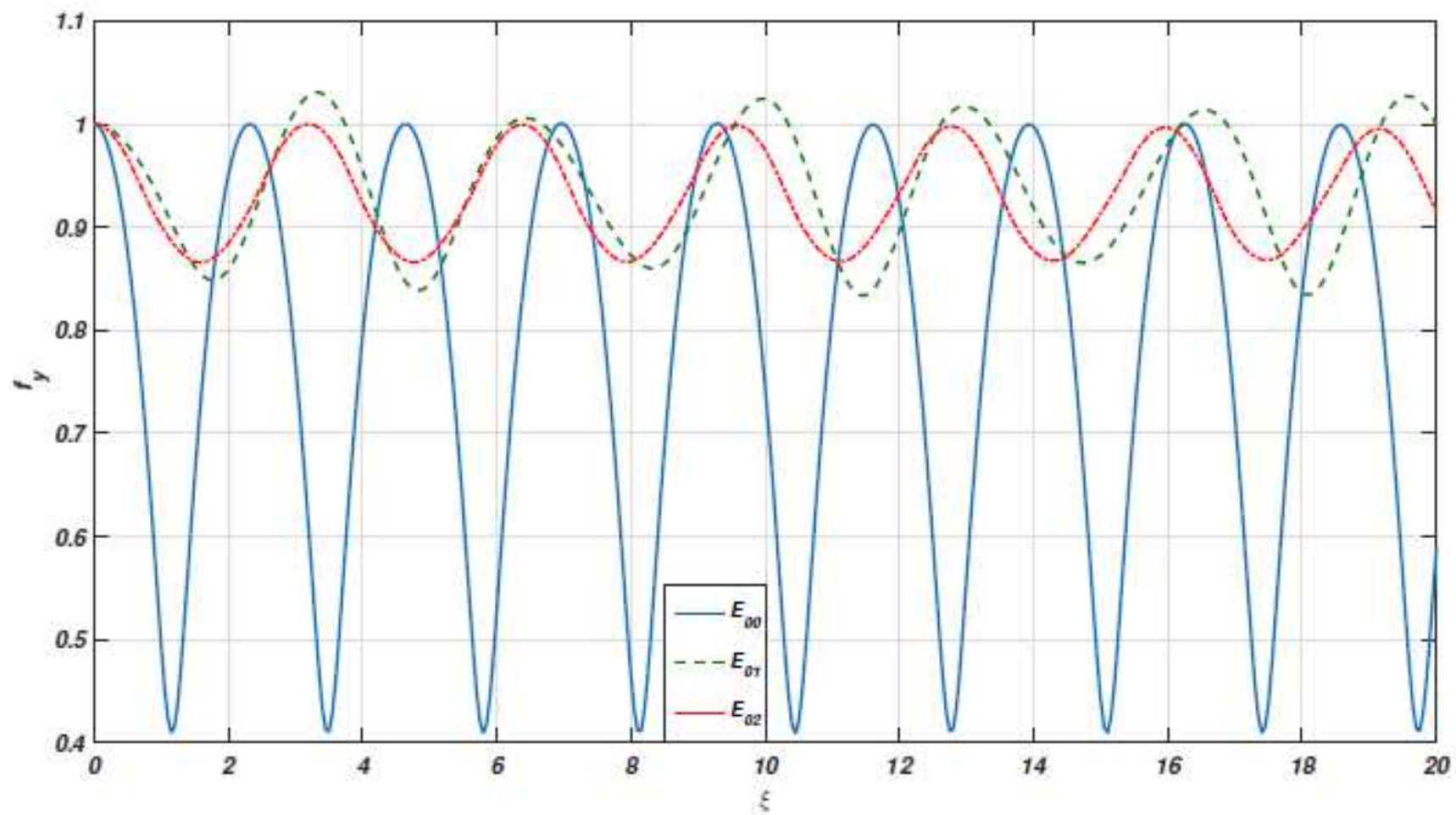

Figure 3 
See the Manuscript Files section for the complete figure caption.

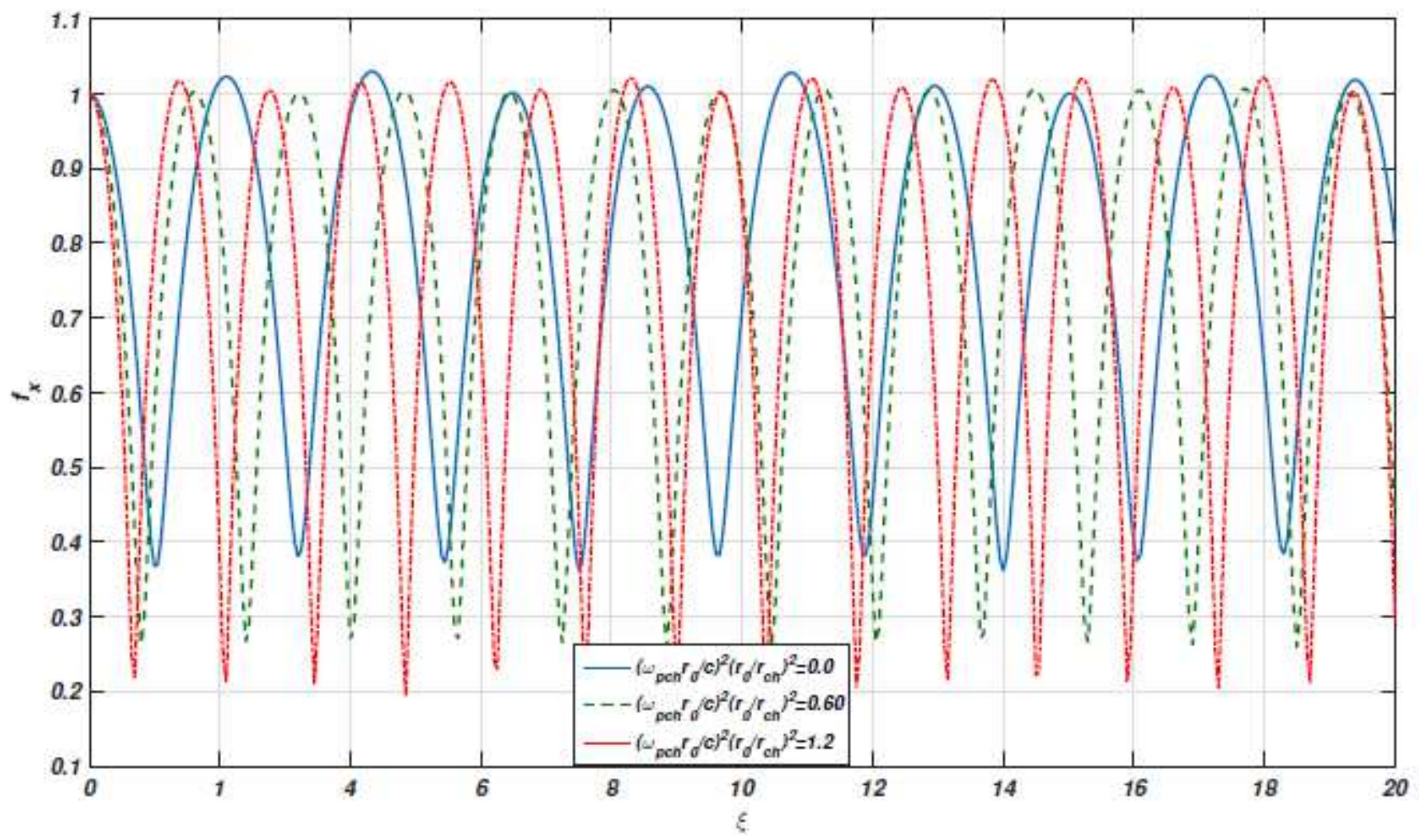

Figure 4

See the Manuscript Files section for the complete figure caption.

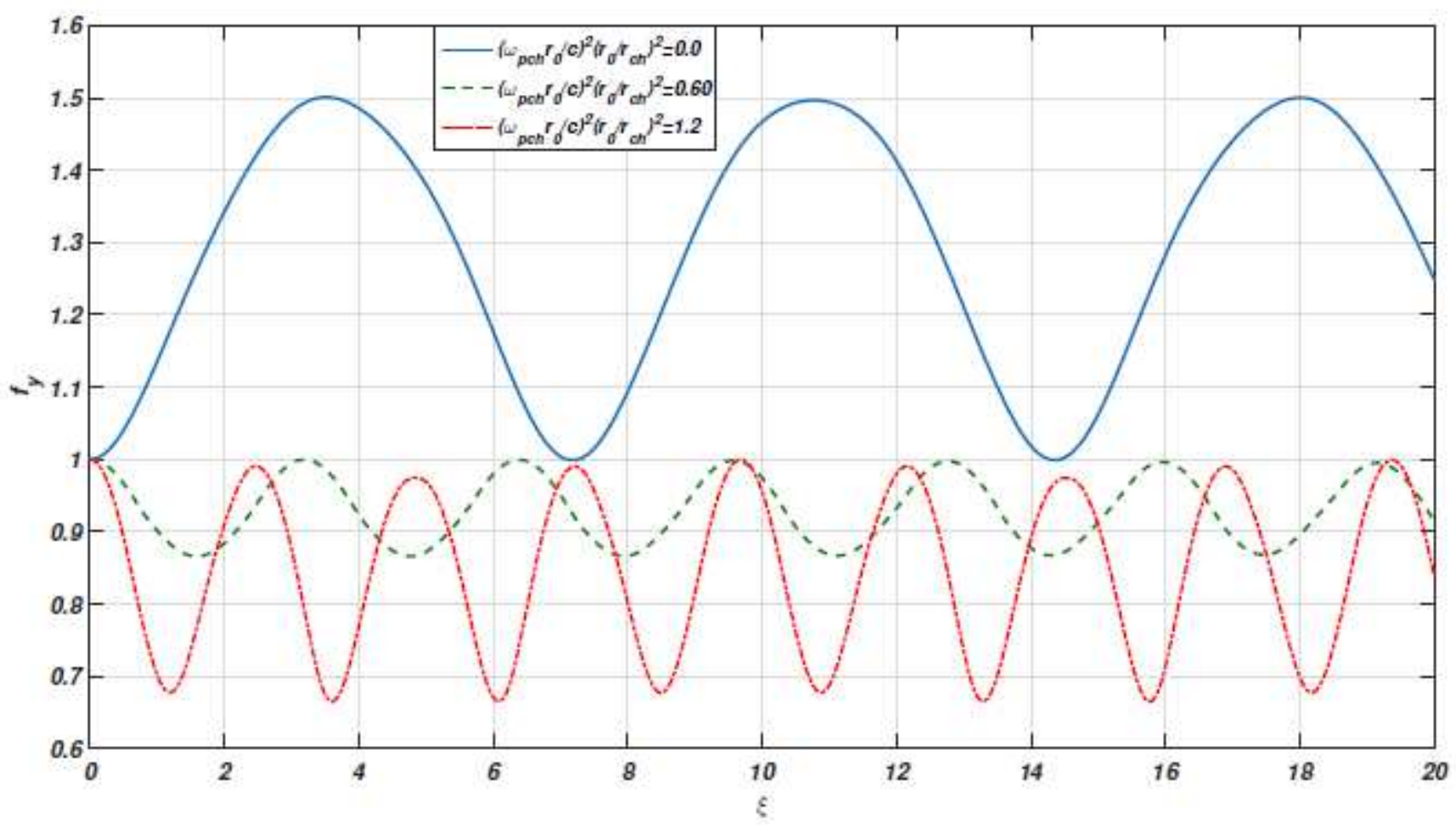


Figure 5

See the Manuscript Files section for the complete figure caption.

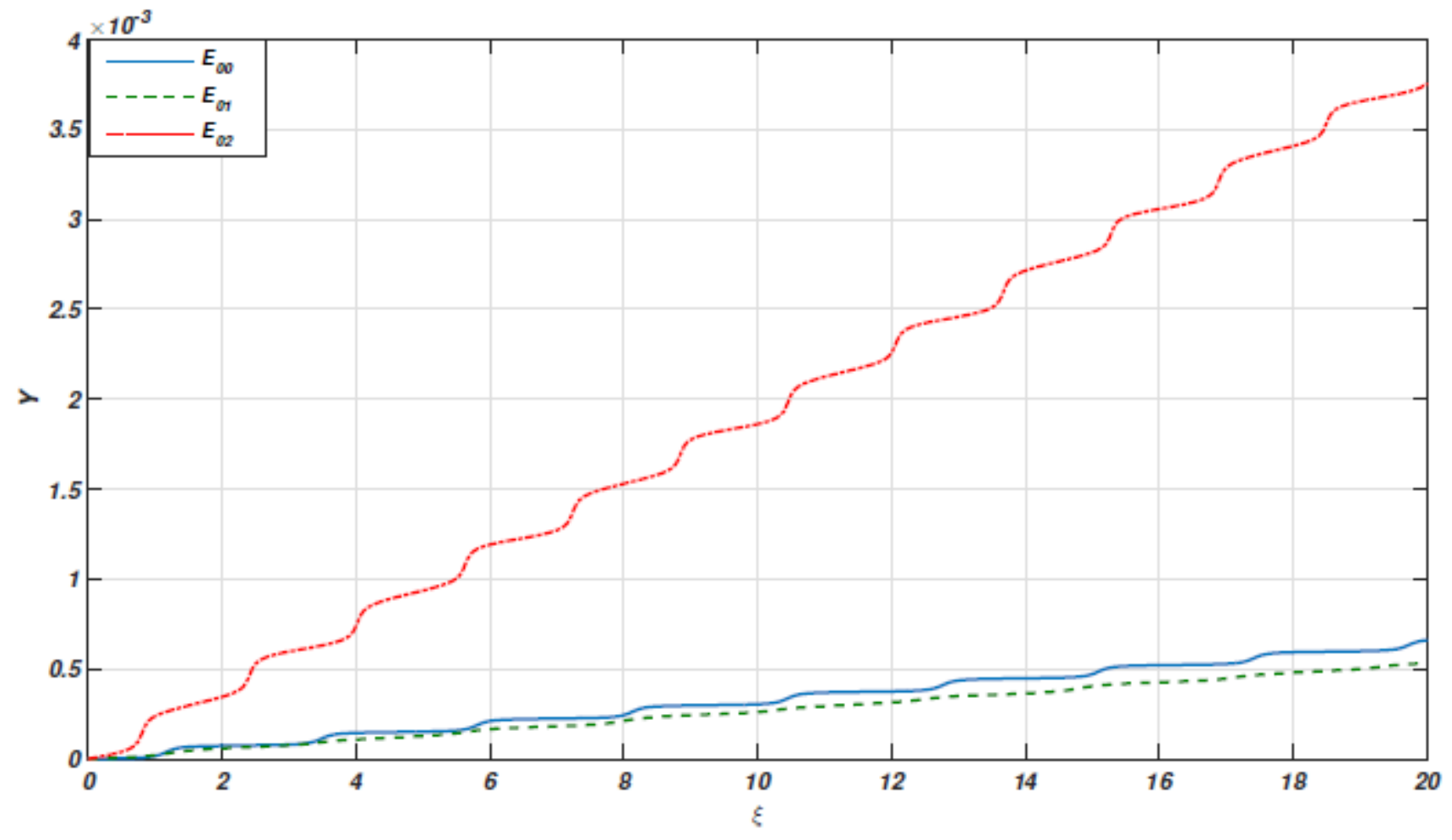

Figure 6

See the Manuscript Files section for the complete figure caption.

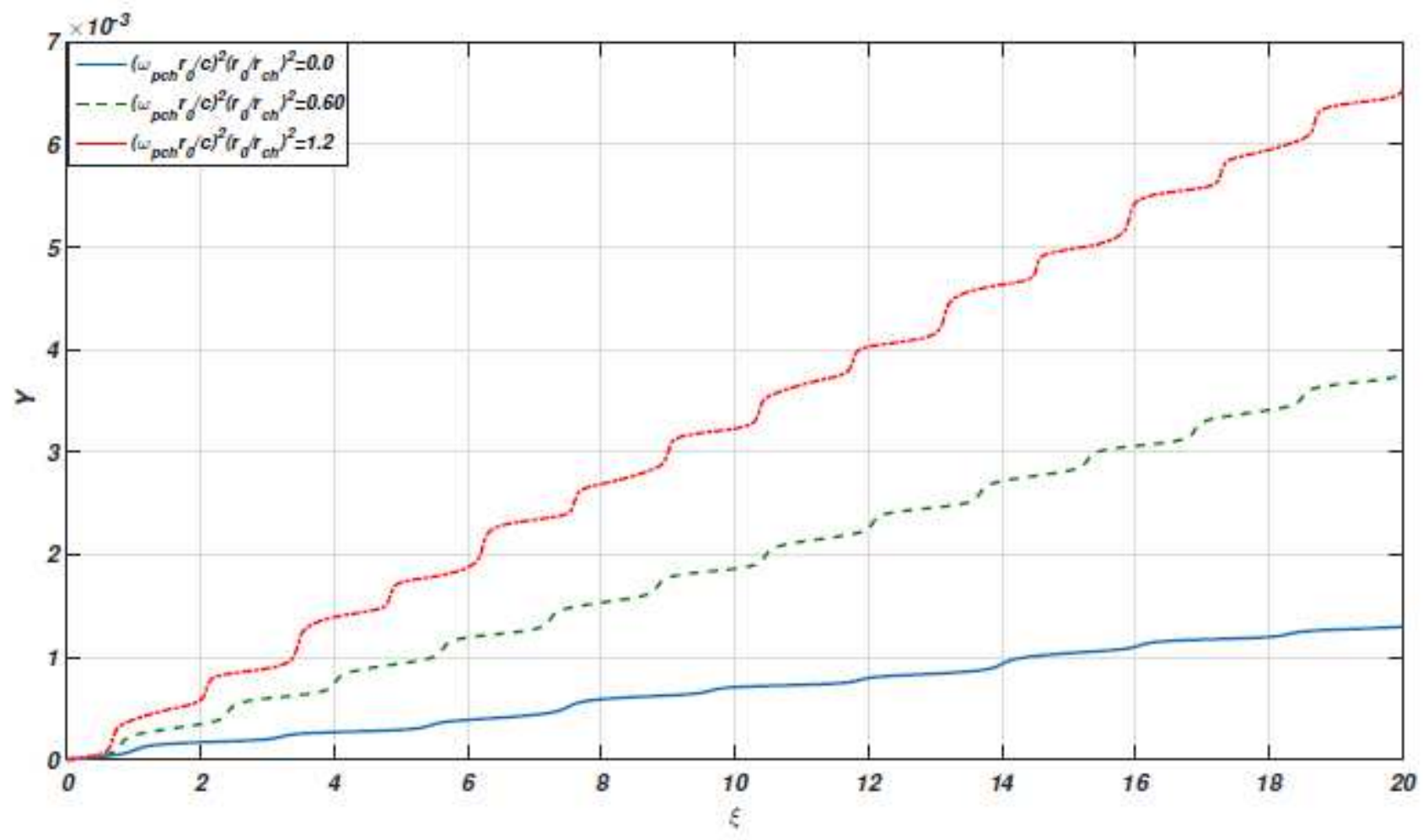


Figure 7

See the Manuscript Files section for the complete figure caption. 